\title{
PROCESS AND SYSTEMS The WRaPT process - $a$ novel and patient-centred approach to workforce planning by a clinically active workforce
}

\author{
Authors: Shalome Kanagaratnam, ${ }^{\mathrm{A}}$ Shruti Dholakia, ${ }^{\mathrm{B}}$ James Wood, ${ }^{\mathrm{C}}$ Alexandra Monkhouse, ${ }^{\mathrm{D}}$ Danny Wood, ${ }^{\mathrm{E}}$ \\ Kavin Abelak ${ }^{F}$ and John Paul Jeans ${ }^{G}$
}

\begin{abstract}
The concept of supply and demand is well established within the health economy of the UK. However, complex health and social care needs, associated with an ageing population, pose a novel challenge to NHS resources and, in particular, its workforce. Although existing strategies adopt a more linear approach to clinical activity and workforce demands, the Workforce Repository and Planning Tool process draws upon the principles of 'realist' data evaluation to combine empirical evidence, practical experience and clinical theory to offer transformation strategies for an NHS workforce that is fit for purpose and its patients.
\end{abstract}

KEYWORDS: Workforce planning, HEE, health and care workforce strategy, WRaPT process, clinical redesign

\section{The problem}

The NHS workforce, approximately 1.4 million people, is undoubtedly its most valuable asset. ${ }^{1}$ This is reflected in its budget, with $65 \%$ of NHS spending allocated towards staffing. ${ }^{2}$ Therefore, the workforce is not only an asset, but also a primary driver of future healthcare costs. Despite this, numerous challenges regarding workforce supply and retention exist, with current figures indicating $45,000 \mathrm{NHS}$ clinical vacancies, $92 \%$ of which are covered by agency or bank staff. ${ }^{2}$ This number of vacancies is projected to worsen in certain staff groups, for example nursing, where the proportion leaving the NHS grew from 7.1\% in 2012 to $8.7 \%$ in 2017 , with 5,000 more nurses leaving the NHS for reasons other than retirement in $2017 .{ }^{2}$ Community services are not immune either; the NHS currently employs $1 \%$ fewer GPs and $26.1 \%$ fewer district nurses than in $2012 .^{2}$ The problem is further

Authors: A emergency medicine specialist registrar, The Royal London Hospital, London, UK; B anaesthetics specialist registrar, University College Hospital, London, UK; ' visualisation lead, $33 \mathrm{~N}$, London, UK; ${ }^{\mathrm{D}}$ anaesthetics specialist registrar, University College Hospital, London, UK; ${ }^{\circ}$ operations lead, 33N, London, UK;

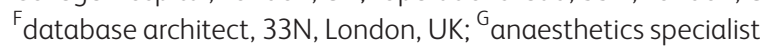
registrar, St Mary's Hospital, London, UK compounded by an ageing population with increasing levels of chronic disease, who place ever more complex demands on health and social care. ${ }^{2}$

Although the Health and Care Workforce Strategy of Health Education England (HEE) means that the NHS workforce should grow by 72,000 by 2027 , this will not meet the predicted demand for 190,000 new staff to deal with the rapidly changing landscape of healthcare. ${ }^{2,3}$

\section{The Workforce Repository and Planning Tool process}

To address the current gap between workforce demand and supply at a local and strategic level, HEE produced the Workforce Repository and Planning Tool (WRaPT). This tool enables a new type of workforce modelling, with the key assumption that changes to the workforce are predicated on changes in activity.

Although WRaPT supports a valuable method of workforce modelling, it has been recognised that using the tool to deliver operationally sound outcomes requires a supplementary process. Therefore, a team of clinically active doctors and data analysts have developed the WRaPT process to support WRaPT in delivering evidence-based workforce transformation that supports individual organisations and departments in the provision of safe, effective patient care.

The steps involved in the WRaPT process (Fig 1) aim to address key challenges facing the future NHS workforce, as outlined by work from The King's Fund. ${ }^{3}$ These challenges include an inappropriate skills mix to match current clinical activity, poor clinical engagement, and substantial data gaps relating to measures of demand and workload. ${ }^{3,4}$ Traditional methods of workforce planning fail to confront these challenges, because a linear relationship between activity and workforce demands is assumed.

Therefore, a primary distinction between the WRaPT process and other methods of workforce planning is the adoption of concepts derived from an emerging approach to data evaluation, known as the 'realist' view. This defines not only the extent, but also the reason behind activity-workforce mismatch. ${ }^{5} \mathrm{~A}$ 'realist' perspective provides a greater understanding of contextual factors, specific to patient group, provider or system, which generates a narrative of service delivery and patient outcomes. ${ }^{6}$ Thus, the WRaPT process combines empirical evidence, practical 


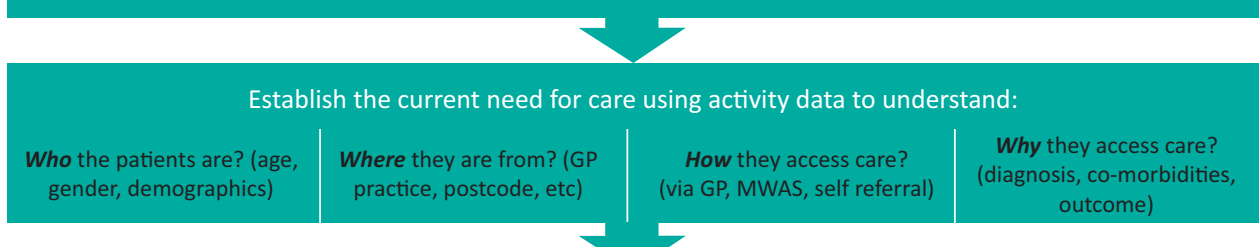

Establish the current workforce baseline:

this will involve collection and validation of the workforce data from across the providers in scope

Fig 1. The Workforce Repository and Planning Tool (WRaPT) process for workforce transformation. $\mathrm{GP}=$ general practioner; $\mathrm{WMAS}=$ West Midlands Ambulance Service; WRaPT $=$ Workforce Repository and Planning Tool.

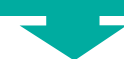

Link the workforce to the activity by engaging with frontline staff, and determine how the workforce links with activity to deliver care and potentially inputting data into WRaPT

Determine how the system/workforce can transform to improve patient care by sharing, promoting and applying best practice and delivering change experience and clinical theory to provide transformation strategies for an NHS workforce that is fit for purpose, while avoiding the increased costs, compromised care quality and clinician disillusion associated with poor clinical redesign. ${ }^{7,8}$

The WRaPT process comprises five stages. It initially involves discussion with system and organisational leads, along with healthcare practitioners, to define the key clinical and/or system question(s). It then combines clinical observation, advanced activity and workforce data analysis using software tools, such as Tableau, Microsoft Excel, SQL and Python, to establish the patient journey, clinical pressures and workforce baseline. At this point, it is possible to identify areas of activity-workforce mismatch and, consequently, opportunities for intervention and transformation.

\section{Case study 1: workforce redesign for Royal Blackburn} surgical triage unit

The surgical triage unit (STU) at the East Lancashire Teaching Hospital has been operational for over 10 years. Since its inception, the unit has grown to incorporate six different specialties and has developed a Hot STU clinic for patients on ambulatory care pathways. Over the past 2 years, the department has recognised increasing operational and workforce pressures on the STU and general surgical wards secondary to high patient volume and workforce gaps. However, evidence of the impact of these pressures on patients and the workforce was not always easily demonstrable. The WRaPT process helped to delineate these pressures into three distinct categories:

$>$ misalignment of the quantity of staff to the volume of activity $>$ a mismatch between the clinical skill mix and the clinical workload $>$ working practices that were not maximally efficient.

Large data sets were then processed and interrogated to identify cohorts of patients according to their length of stay, severity of diagnosis and hospital journey (Fig 2). In this case, although all wards were staffed equally, Ward C22 had a higher volume of patients passing through, thereby generating more work for staff. This was compounded by a long admissions process for elective
Fig 2. Patient flow for direct referrals and indirect referrals (via accident and emergency; A\&E) to surgical triage unit for all surgical attendances with an A\&E or general practitioner referral source from 01

February 2016 to 30 July 2017. $\mathrm{A} \& \mathrm{E}=$ accident and emergency department; $C R I C=$ critical care; HOTSTU $=$ hot surgical triage unit; SADU = surgical admissions and day unit; STU = surgical triage unit.

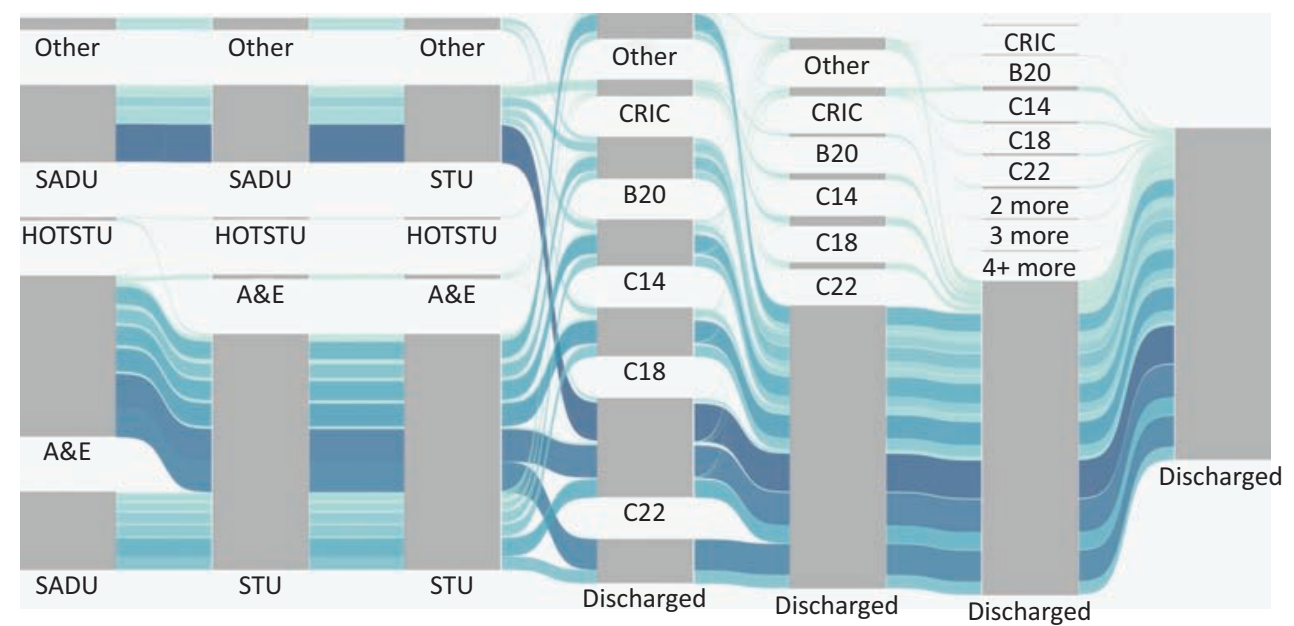




\begin{tabular}{|c|c|}
\hline Diagnosis severity & Percentage of attendances to the UCC \\
\hline Minor & $42,333(52.5 \%)$ \\
\hline Moderate & $2,797(3.5 \%)$ \\
\hline Severe & $7,717(9.6 \%)$ \\
\hline Variable & $25,372(31.4 \%)$ \\
\hline Unspecified & $2,476(3.0 \%)$ \\
\hline
\end{tabular}

patients. Patients were thereafter streamlined into those likely to be discharged within 4 days and those requiring a longer length of stay.

It was recommended in the redesign that those complex patients with a greater length of stay should be admitted directly to a general surgical ward to avoid unnecessary duplication of work and patient transfers, all of which contributed to a greater overall length of stay. Meanwhile, short-stay patients could be concentrated in the STU for clerking and management by junior doctors.

These recommendations translated to adjustments in workforce distribution to include a greater consultant presence in accident and emergency (A\&E) and STU to enable quicker decisionmaking and admission avoidance where appropriate. The role of foundation doctors would have greater emphasis on clerking to support consultants, while a cohort of physician associates would manage the general surgical wards to streamline the convalescence and discharge of long-stay patients. This would leave core and higher trainees more clinic and theatre time to fulfil training needs. Thus, increased learning opportunities for junior doctors were an important by-product of this proposed solution and highlight how workforce transformation is about delivery not only of activity, but also of training. Other recommendations included augmenting existing patient pathways and removing processes that added no value to long-stay patients. These recommendations will support the trust in shifting appropriate activity out of the hospital, reducing the length of stay and reducing pressure in $A \& E$.

Case study 2: workforce redesign in the A\&E of an acute hospital

Emergency care in an acute trust in the midlands is currently considering a significant reconfiguration in response to increasing operational pressures. This reconfiguration involves consideration

Table 2. Complexity of primary investigations and treatments within the urgent care centres

\begin{tabular}{|c|c|c|}
\hline \multirow[t]{2}{*}{ Complexity } & \multicolumn{2}{|c|}{ Frequency and percentage within the UCCs } \\
\hline & Primary investigations & Primary treatments \\
\hline Simple & $46,521(57.7 \%)$ & $50,067(62.0 \%)$ \\
\hline Intermediate & $34,071(42.2 \%)$ & $24,828(30.8 \%)$ \\
\hline Complex & $101(0.1 \%)$ & $5,795(7.2 \%)$ \\
\hline Null & $2(0.0 \%)$ & $6(0.0 \%)$ \\
\hline
\end{tabular}

Table 3. Most frequent complex investigations and treatments for attendances streamed by the WRaPT algorithm to urgent care centres

\begin{tabular}{lll}
$\begin{array}{l}\text { Investigation or } \\
\text { treatment group }\end{array}$ & $\begin{array}{l}\text { Investigation or } \\
\text { treatment }\end{array}$ & $\begin{array}{l}\text { Number of } \\
\text { attendances }\end{array}$ \\
$\begin{array}{l}\text { Complex investigation } \\
\text { Complex treatment }\end{array}$ & $\begin{array}{l}\text { Ultrasound } \\
\text { CPAP/nasal CPAP/bag } \\
\text { valve mask ventilation }\end{array}$ & 2,783 \\
& Arterial line & 1,938 \\
& Incision and drainage & 300 \\
& Manipulation of dislocation & 262 \\
\hline
\end{tabular}

$\mathrm{CPAP}=$ continuous positive airways pressure; $\mathrm{UCC}=$ urgent care centre; WRaPT = Workforce Repository and Planning Tool.

of a 'hot site' with an A\&E and urgent care centre (UCC) and a 'cold' site with a UCC only. Historical data from a 30-month period were processed relating to patient demographics, mode of presentation, diagnosis and treatment codes, and length of stay in the department. These data was analysed using the WRaPT process and revealed that the current algorithm for patient streaming would mean that the UCC would see patients more complex than initially expected (Tables 1-3).

Further work by WRaPT helped to refine the algorithm to ensure that patients could be appropriately, and safely, streamed to either UCC or A\&E. An activity-matched workforce was thereafter derived using Workforce Dashboards (Fig 3) that enable the user to create new roles and investigate their impact within the department according to patient acuity and the complexity of investigations and treatments. These Workforce Dashboards are being incorporated into an updated version of WRaPT.

Finally, interrogation of the data also demonstrated that greater occupancy of the department correlated with poor performance. Occupancy was influenced most significantly by age of the patient population present within the department. As such, the inclusion of a rapid specialist assessment service and frailty unit at the front door was recommended as a method of shortening length of stay and avoiding unnecessary admissions in the older patient population. Other proposals sought to transfer non-urgent activity away from the main department and included the introduction of dedicated streaming for minor injuries, through emergency nurse practitioners or extended-role physiotherapists, and fostering links with GP practices of high usage to establish and reinforce community care pathways.

\section{Benefits and limitations}

The integration of observations of working practice and a focus on clinical need highlight a systems approach that looks along and across patient pathways. The proposed reconfigurations, although perhaps not ground-breaking in themselves, are derived from a model that looks beyond arbitrary numbers to the nature of clinical activity and, as such, ensures that workforce planning is driven by both a qualitative and quantitative understanding of clinical activity to ensure optimal patient care.

Furthermore, the above case studies were performed in the context of wider system transformation. This means that, although the use of the process delivered operationally relevant 
Fig 3. Workforce staffing model demonstrating how analysis of which staff groups undertake which types of clinical activity can be used to support the addition of advanced clinical practitioners in a new emergency centre. This dashboard is currently being worked into an updated version of the Workforce Repository and Planning Tool.

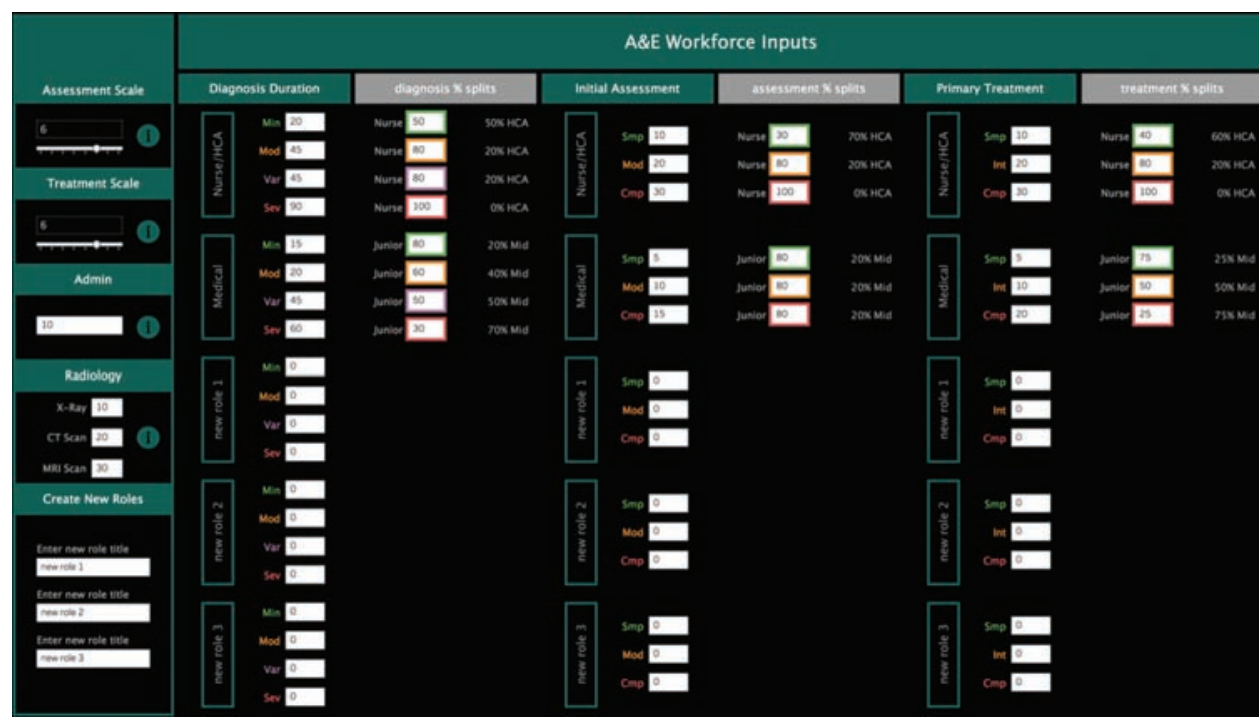

recommendations, it informed wider thinking regarding system transformation. For example, the first case study highlighted how outlying non-surgical patients, theatre flow and A\&E admissions need to be considered when redesigning the surgical workforce. This prompted further use of WRaPT in A\&E and acute medicine to support the redesign of clinical pathways, thereby helping the trust with performance targets in areas with chronic workforce shortages.

However, the WRaPT process is in its early stages and, therefore, comparative work with traditional strategies for workforce planning is yet to be performed. Furthermore, the process is wholly reliant on the interrogation of good-quality data. As such, departments are required to ensure that clinical coding and data collection are accurately collated on central IT systems to yield a true reflection of clinical need. Therefore, training in the systems approach and data analytics is needed among practicing clinicians and is an exciting area for further work.

Looking forward, we hope that more clinicians and allied healthcare professionals will use the WRaPT process to make the important, granular associations required for workforce planning. We also hope to trial the process in various clinical environments, such as primary care, community care and social care. This will become particularly pertinent given the current drive towards admission avoidance in the NHS.

\section{Conclusion}

The WRaPT process marks an important and unique step in NHS workforce planning. Although organisations routinely collect large quantities of data, the real challenge lies in the comprehensive and clinically relevant interrogation of these data. Through the inclusion of a clinically active workforce in this process, the nuances of service inefficiencies and activity-workforce mismatch could be better understood. Furthermore, clinically active practitioners are well placed to engage with front-line staff to obtain insights into the optimisation of the current service provision. This creates an open and honest sounding-board that not only informs proposals, but also ensures that existing healthcare practitioners are empowered by the transformation process.
By illustrating these findings through advanced visualisation tools, the WRaPT process ensures that conclusions are both accessible and meaningful to the NHS and social care in an entirely new way. This facilitates collaboration and builds in-house capabilities for dynamic workforce redesign. The subsequent outcome is one of evidence-based workforce remodelling and system transformation that are achievable and sustainable for NHS staff and, most importantly, for their patients.

The WRaPT tool can be accessed at https://thisis.wrapt.org.uk/ home and is free to use for NHS and social care providers. Further enquires on the WRaPT process should be directed to wrapt@ lancashirecare.nhs.uk

\section{References}

1 NHS Confederation. NHS statistics, facts and figures. NHS, 2017. www.nhsconfed.org/resources/key-statistics-on-the-nhs [Accessed 19 December 2018].

2 Public Health England. Facing the facts, shaping the future: a draft Health and Care Workforce Strategy for England to 2027. London: Public Health England, 2017.

3 The King's Fund. Workforce planning in the NHS. London: The King's Fund, 2015

4 Authority of the House of Lords. The long-term sustainability of the NHS and adult social care. London: House of Lords, 2017.

5 Malone J, McCormack B, Hutchinson A et al. Realist synthesis: illustrating the method for implementation research. Implement Sci 2012;7:33.

6 Pawson R, Greenhalgh T, Harvey G, Walshe K. Realist review - a new method of systematic review designed for complex policy interventions. J Health Serv Res Policy 2005;10(Suppl 1):21-34.

7 Bohmer R, Imison C. Lessons from England's health care workforce redesign: no quick fixes. Health Affairs 2013;32:2025-31.

8 Hyde P, McBride A, Walshe K, Young R. A catalyst for change? The National Evaluation of the Changing Workforce Programme. Manchester: Manchester University Business School, 2004.

Address for correspondence: Shalome Kanagaratnam, 33 Northstand Apartments, Highbury Square, London N5 1FJ, UK. Email: shalomekanagaratnam@nhs.net 